\title{
Absorption, Distribution, Metabolism and Excretion of Tattoo Colorants and Ingredients in Mouse and Man: The Known and the Unknown
}

\author{
Wolfgang Bäumler \\ Department of Dermatology, University of Regensburg, Regensburg, Germany
}

\begin{abstract}
During tattooing, high amounts of tattoo colorants, which usually contain various substances, are injected into skin. The major ingredient in tattoo colorants is the coloring component, which can be assigned to two different groups. First, amorphous carbon particles (Carbon Black) are found almost exclusively in black tattoos. Second, tattooists use azo and polycyclic pigments to create nearly all colors of the visible spectrum. Due to their different chemistries, those tattoo colorants usually contain various compounds, such as by-products and impurities. Professional tattooists inject the colorant mixture into skin using the solid needles of tattoo machines, and studies have shown that about $2.5 \mathrm{mg}$ of tattoo pigment is injected to stain about $1 \mathrm{~cm}^{2}$ of skin. Animal experiments revealed that about one-third of that amount disappeared from skin within weeks after tattooing, and this finding was confirmed by pigment extraction from longexisting tattoos. It is assumed that some of the tattoo colorants stay in the skin because the pigment particles are insoluble and too large to be transported. The other part
\end{abstract}

of the tattoo colorants shows up at least in the lymph nodes located next to the tattoo. To date, no investigations determining whether and to what extent tattoo colorants can be found in any other organs of the human body have been performed. Thus, tattooing of colorants into skin entails a complex reaction of the skin that triggers the immune system and launches manifold transport processes, which might pose additional health risks not only to skin but also to other organs of humans.

(C) 2015 S. Karger AG, Basel

\section{Introduction}

Tattooing is an ancient technique used to stain the skin of humans. Tattoos have been identified in human beings dating back to the Stone Age [1] In some cultures, like Polynesian tribes, tattooing was an important tool in religion and hierarchy. However, the meaning of tattoos in the western world became ambiguous during the past 
centuries and was consistently associated with low social status.

Nowadays, tattooing has become very popular worldwide. The current tattoos are black or multicolored and can be found on almost all parts of the human body. Many tattooed role models, like football, pop or movie stars, have led to a broader cultural acceptance of tattoos. A special category of tattoos is the so-called permanent make-up, in which colorants are placed in the skin to mimic normal make-up [2].

Tattooists usually inject colorants as a suspension into the skin using solid needles, which are actuated by tattoo machines. Tattooists purchase black colorants from tattoo suppliers or through the internet. A fraction of the injected colorant stays in the dermis as particles, and their light absorption within a specific spectral range results in the color of the tattoo. Another fraction of the injected colorant is removed from the skin via the lymphatic or blood vessel systems. As a result, tattoo colorants can be found in the lymph nodes located next to the tattoo [36]. An internet-based survey showed that about $60 \%$ of tattoos are either completely or partly black [7].

\section{Chemistry of Tattoo Colorants and the Related Health Concerns}

From a chemical point of view, colorants are classified as either pigments or dyes; however, the chemical structures of pigment and dye molecules are frequently the same. In contrast to dyes, pigments are practically insoluble in the medium in which they are incorporated, and this insolubility is achieved by avoiding solubilizing groups in the molecules, yielding small particles, the socalled pigments. Pigments may be inorganic or organic, colored, white or black materials.

Thus, making a persistent tattoo in skin requires the use of water-insoluble colorants in the form of pigments. In the past, tattooists used in- organic pigments that contained heavy metals such as mercury, chromium or cadmium, resulting in the typical colors yellow (cadmium sulfide), mercury sulfide (red), or chromium oxide (green). Two important inorganic pigments are still in use: Carbon Black for black tattoos and titanium dioxide to reduce the color strength of colored pigments.

Nowadays, colored tattoo colorants mainly consist of organic pigments like azo or polycyclic pigments, which are usually obtained from the chemical industry [8]. These pigments comprise two features perfect for use in tattoos: the pigments exhibit brilliant colors, and they are insoluble in aqueous tissue. The chemistry of black inks, which predominantly contain Carbon Black, has not changed over time. Carbon Black is a powder that mainly comprises amorphous particles of carbon with diameters of a few tenths of nanometers (fig. 1).

Although they are injected into the human body, tattoo colorants usually have no pharmaceutical requirements. Beside the coloring compound (black, red, green, blue, etc.), the colorants may contain other various substances depending on the production methods. The colorants comprise educts, products, and by-products of the respective coloring compound [9]. Different solvents (water, isopropanol, etc.) are used to dissolve the pigment powder, other substances are applied as preservatives, and titanium dioxide is frequently added to change the color strength $[8$, 10]. Tattoo colorants might also contain various impurities that accidentally got into colorants for unknown reasons. Thus, tattoo colorants usually exhibit a complex mixture of various chemical compounds [11-13]. At present, the list of identified admixtures and impurities is rather incomplete, and further chemical analysis of the tattoo colorants that are present in the market is required. It is known that the chemistries for colored and black tattoo colorants are rather different, and hence, the colorants may contain different by-products and impurities $[8,11]$. 

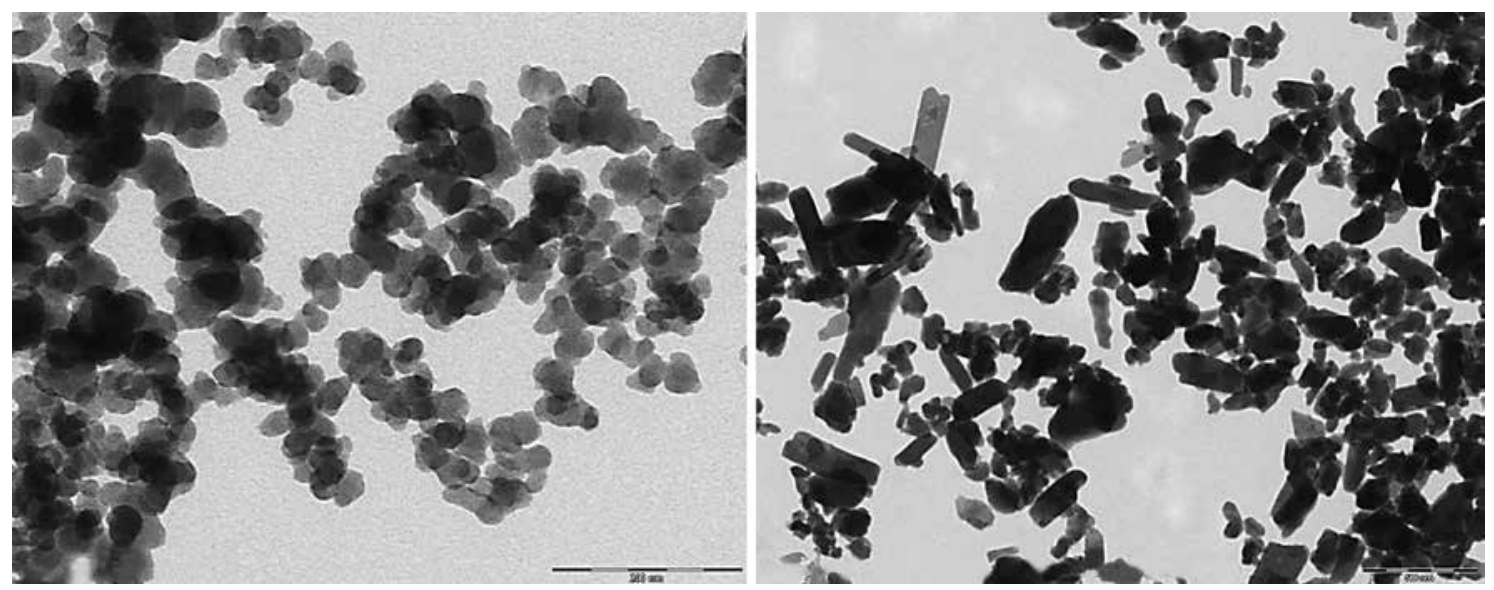

Fig. 1. Electron microscopy images showing the details of commercially available tattoo colorants. The black colorant is comprised of rounded particles (left), whereas red azo pigments (PR22) have a more elongated shape (right). The mean diameter of the particles is in the range of several tenths of nanometers.

\section{Colored Pigments}

Colored pigments are classified by their chemical constitution and can be roughly assigned to azo or polycyclic pigments. Pigments are identified either by their chemical index number or by the pigment shortcut. The azo pigments are subdivided in mono-azo (greenish to medium yellow, reddish yellow to orange), dis-azo (greenish, reddish to orange red), $\beta$-naphtol (orange to medium red), naphtol AS (medium red to violet) and metal complex pigments containing nickel, copper or cobalt. The polycyclic pigments are generally condensed aromatic or heterocyclic ring systems. Two important examples are the phthalocyanines (green, blue) and the quinacridone pigments (bluish red, red, violet) [8]. The production of such pigments requires complex chemical synthesis, and the resulting colorant may contain different educts, products and by-products as well as titanium dioxide, to lighten the colorant, and different concentrations of other non-specified compounds.

Colored tattoo pigments like PR.22 can be decomposed by solar radiation $[14,15]$ or by laser light [16], yielding decomposition products such as 2-methyl-5-nitroaniline (2,5-MNA), 4-nitrotoluene (4-NT), 2,5-dichloroaniline (2,5-DCA)

and 1,4-dichlorobenzene (1,4-DCB) (fig. 1). 4-NT was genotoxic in a human lymphocyte assay [17], and 2,5-MNA, also referred to as 5-nitro-o-toluidine, was shown to cause liver dysfunction in workers from a hair dye factory [18]. Additionally, Sayama et al. [19] showed that 2,5-MNA and some dinitrotoluenes are mutagenic to Salmonella typhimurium YG. Furthermore, 1,4-DCB induced kidney tumors in male rats and liver tumors in male and female mice [20], whereas 2,5-DCA was nephrotoxic in rats [21].

\section{Black Colorants}

Black colorants are usually produced by the imperfect combustion of hydrocarbons, yielding soot with polycyclic aromatic hydrocarbons (PAHs). The major constituent of black tattoo colorants is Carbon Black, which has been listed by the International Agency of Research in Cancer (IARC) as possibly carcinogenic to humans (group 2B) [22]. High amounts of hazardous PAHs, up to $201 \mu \mathrm{g} / \mathrm{g}$, were found when analyzing different commercially available black tattoo colorants [11]. PAH molecules are either unbound in the colorant suspension or are attached to the surface of Carbon Black particles. PAHs such as 
benzo[a]pyrene belong to a large class of wellstudied chemical pollutants that ubiquitously occur in the environment. They consist of two or more fused benzene rings and are generated naturally or are found as a result of incomplete combustion of organic materials, fossil fuels, vehicular emissions or even tobacco smoke. Some PAHs are classified by the IARC as human carcinogens (e.g., benzo[a]pyrene), and several others are classified as probably or possibly carcinogenic to humans [23]. The active metabolite benzo[a]pyrene-7,8-diol-9,10-epoxide probably represents the ultimate carcinogen [24].

It is well known that human exposure to complex mixtures of PAHs occurs primarily through three routes: the respiratory tract through the smoking of tobacco products and the inhalation of polluted air, the gastrointestinal tract through the ingestion of contaminated drinking water and food, and skin contact, which usually occurs from occupational exposure [25].

In contrast to topical coal tar application and possible penetration into skin, black colorants are injected into skin during the making of a black tattoo, resulting in an almost complete penetration of PAHs into the skin, after which the substances can distribute throughout the entire body. In addition to their carcinogenic properties, PAHs exert a wide range of deleterious effects towards tissue and cells, including the mutagenesis of oncogenes in skin [26]. PAHs are thus potent immunotoxic agents that impair the functional activation of lymphocytes [27] and inhibit macrophage differentiation [28]. Other analytical investigations revealed the presence of additional hazard substances, such as the softener di-buthyl-phthalate [29]. Another problem may arise from the fact that the particles have small diameters of tenths of nanometers [30]. Thus, the potential of the particles for nanotoxicology should be investigated [31].

Consequently, the skin might react to either colored or black ink suspensions in different manners. Many side effects of tattoos, such as granulomatous, lichenoid or hypersensitive aller- gic reactions, infections, and malignant skin tumors, have been described in the medical literature $[2,12,13,32]$; however, the latter might be coincidental [33].

\section{Tattoo Colorants in Skin}

In the western world, most tattooists inject tattoo colorants into skin using the rapidly vibrating needles of a tattoo machine to transport a certain amount of colorant suspension into the skin. The needles thereby leave punctures in the skin that pierce the thin epidermis and may reach the middle of the dermis (fig. 2, left). Experiments with pig and human skin revealed the concentration of, e.g., red pigment, that is placed in skin by such tattoo machines. As tattooing is an archaic procedure, the experiments yielded a concentration range of about $0.60-9.42 \mathrm{mg}$ of pigment per $\mathrm{cm}^{2}$ of tattooed skin [34]. The concentrations depended on the size of the pigment crystals, the concentration of pigment applied to the skin surface, the desired color strength of the tattoo, and the tattooing procedure using needles of different sizes and shapes. The mean value was estimated to be about $2.5 \mathrm{mg} / \mathrm{cm}^{2}$.

Tattooing damages the skin, causing pain and superficial bleeding. In an internet-based survey, tattooed people reported crust formation, itching, swelling and even superficial infections during the healing process [7]. Being a superficial injury to the skin, the tattooed area should heal within a couple of days. However, the survey also revealed that $8 \%$ of the participants still had health problems 4 weeks after tattooing, and 6\% had persistent skin problems in the tattooed area. Additionally, 3\% stated other health problems such as psychiatric problems and light sensitivity of the tattooed skin. These problems were significantly related to the color of the tattoo, which obviously meant that they were related to the specific chemistry of the colorant. This finding was confirmed by comparing the data of that survey with medical case reports 

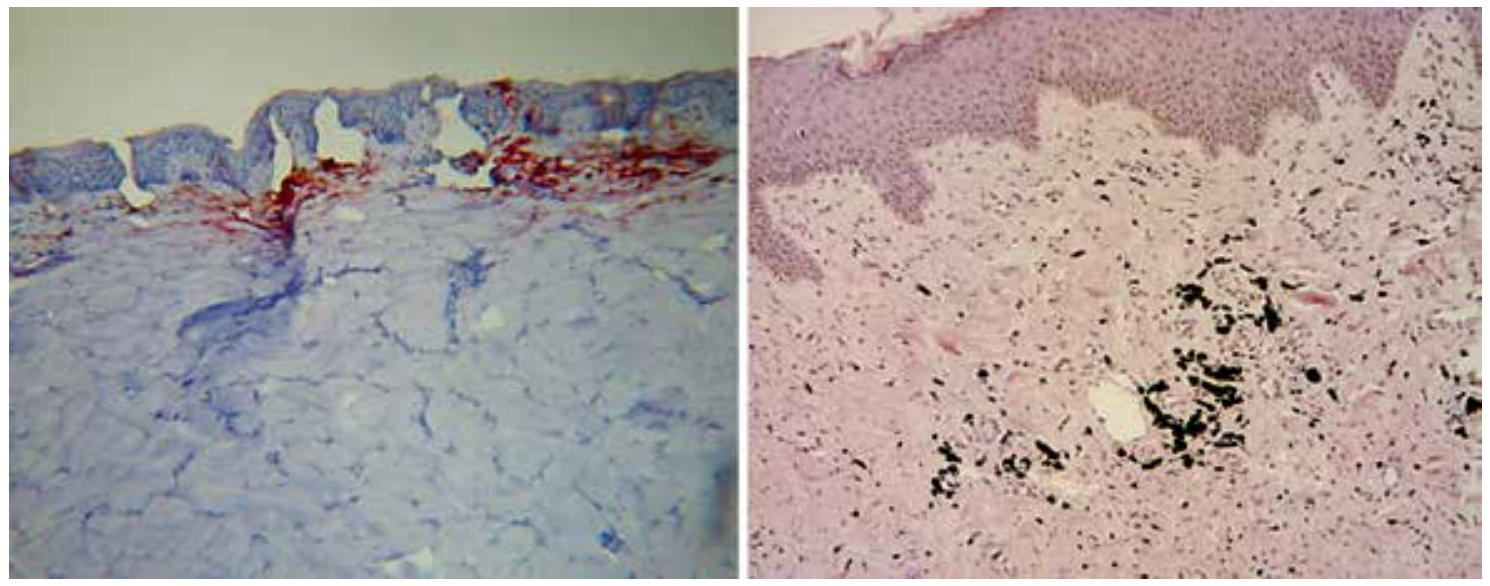

Fig. 2. Pig skin tattooed with a red-colored pigment (left). The histology shows the color in the superficial dermis and that the tattooing needles left holes in the skin. A punch biopsy was taken from black-tattooed skin (right). The histological slice shows black particles in the dermis. Only large agglomerates of pigments can be detected in the histology using light microscopy at a spatial resolution of about $0.5 \mu \mathrm{m}$. Regardless of the place of injection, the pigment particles move to different sites within the dermis by carriers such as macrophages.

on the location and color of tattoos. The results clearly showed that colored tattoo pigments, in particular red pigments, are mainly responsible for adverse skin reactions and that these reactions occur more often on the extremities $[12,13]$.

After tattooing skin, pigment particles are exclusively found in the cytoplasm of cells in membrane-bound structures identified as secondary lysosomes [35]. Also, macrophages may contain pigment particles. At first view, the injected tattoo colorants seem to stay in skin forever. However, three major mechanisms may reduce the concentration of colorants that is initially placed in the skin. First, part of the colorant may leave the skin with the bleeding during or directly after tattooing. Second, part of the colorant may be transported away from the skin via the lymphatic or blood vessel system. Third, part of the colorant is decomposed months or years after tattooing because the pigments in the dermis are repeatedly exposed to different light sources, in particular, solar radiation, including UV radiation. Azo pigments are chemically unstable when exposed to UV radiation [14, 15].
Recent investigations provided evidence that the major part of tattoo colorants, such as red pigments (87-99\%), disappears from skin months or years after tattooing [36], which could cause a fading of the colored skin. However, when asking tattooed people, almost nobody perceives a change in the tattoo color [7]. The change in the color concentration can be overlooked because of the very high color strength of azo pigments. The decrease in the pigment concentration appears to be very high; however, an in vivo animal model showed that about $30 \%$ of intradermally injected PR 22 disappeared from skin within 6 weeks after tattooing [37], and up to $60 \%$ of tattooed PR22 disappeared when applying excess solar radiation to the animal skin [37]. Thus, the disappearance of such a high fraction of the tattooed pigments is a result of either light-induced decomposition in tattooed skin or pigment transport to other anatomical locations in the body via the lymphatic system.

For example, black tattoo colorants frequently contain substantial amounts of PAHs [11], and enzymatic and non-enzymatic pathways can convert PAHs into hazardous diol-epoxides, 
such as benz[a]pyrene-7,8-diol-9,10-epoxide [38]. Both pathways might also occur in tattooed skin. First, cytochrome P450-dependent enzymes can be triggered by injecting foreign material, such as tattoo ink, into skin [39]. Second, PAHs in tattooed skin generate singlet oxygen when exposed to UV radiation, leading to oxidation of the PAHs. Akintobi et al. showed the induction of cytochrome P450 1B1 (CYP1B1) expression in human dermal fibroblasts when exposed to xenobiotic substances like 2,3,7,8-tetrachlorodibenzo-p-dioxin [40]. Recent studies have shown that CYP1B1, a newly identified member of the CYP1 family, plays a very important role in the metabolic activation of PAHs $[41,42]$. Whether such cytochrome P450-dependent enzymes also play a role in tattooed skin has not been investigated.

Thus, educts, by-products, impurities, and admixtures can be punctured into skin to an unknown extent during tattooing. These compounds may cause various adverse skin reactions, which have been consistently published in the literature $[12,43]$. Also, case reports about skin tumors from tattoos have been published and summarized in a recent review article [33]. However, it is still under discussion whether the malignancies, including basal cell carcinoma [44] or malignant melanoma, were coincident.

\section{Transport of Tattoo Colorants to Other Anatomical Sites}

Tattoo colorants are a rather complex mixture of various compounds that exhibit different chemical and physical structures. The colorants contain pigment particles of different sizes and molecules, and many of the molecules are in the form of monomers, dimers, or polymers with different solubilities, which influences the extent and the route of transport inside the dermis and to other organs. In addition to transport, some constituents of the colorants can be metabolized in skin or

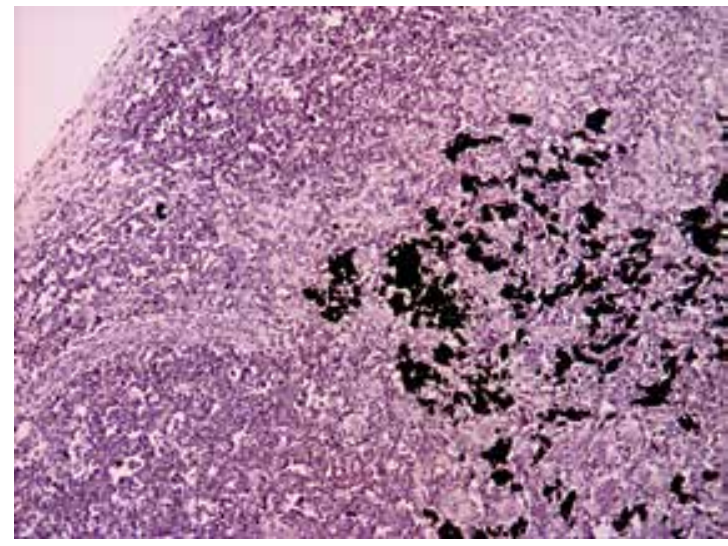

Fig. 3. A histological slice showing black colorant particles in a lymph node located in the axilla.

in the organs to which the compounds were transported. Thus, tattooing of colorants into skin entails a complex reaction of the skin that triggers the immune system and launches manifold transport processes.

After injection into skin, some of the tattoo pigment particles are encapsulated in the dermis. As mentioned above, the colorants can be transported to other anatomical locations in the body via the blood vessel and lymphatic systems directly after tattooing or via the lymphatic system years or months after tattooing. In particular, a portion of the small particles, admixtures, impurities, as well as educts and products of the pigment molecules may leave the skin directly or during the weeks after tattooing. These transportable compounds have the potential to reach other anatomical locations and might be stored in other organs or may leave the human body via the urinary system or defecation. Thus, organs such as liver, spleen and kidney could be destinations of constituents of tattoo colorants, depending on the route of transport via the lymphatic or blood vessel systems. Consequently, after tattooing the skin, the injected colorants may pose a risk to other organs in the human body. However, except for the transport of tattoo colorants to the lymph nodes (fig. 3) [3, 4, 45-48], all other transport 
processes and routes in a human body have been unexplored.

It is assumed that especially large particles, which cannot pass the lymph nodes, stay in the dermis. Thus, any process that reduces the size of the particles will assist in reducing the concentration of pigment particles in skin. A major mechanism for disintegration of the particles is the light-induced decomposition of pigment molecules that may continuously occur whenever tattooed skin is exposed to light sources [14]. Other mechanisms, like enzymatic activities or the recurring activation of macrophages, might contribute to pigment particle transport. However, the contribution of other mechanisms is unknown.

There are currently no indications regarding the systemic effects of tattoo colorants and of their decomposition products because scientific investigations or epidemiologic data are lacking. Millions of people have many and large tattoos [7], with sizes of $600 \mathrm{~cm}^{2}$ and more. In cases of such tattoos, about 1,500 mg of, e.g., azo pigments are injected into the human body. In light of the $80 \%$ decrease, one should deliberate about whether 1,200 mg of azo pigments and their possible decomposition products could cause health problems in the skin or in the rest of the human body.

In the case of black colorants, substantial amounts of PAHs are injected into skin and hence should be partially transported to other organs.
This is of great importance because those PAHs might cause deleterious effects elsewhere in the human body. Recently, placental concentrations of PAHs were analyzed by gas chromatographymass spectrometry. PAH concentrations above the median, as detected in some cases, were associated with a 4.52-fold increased risk for neural tube defects, a 5.84-fold increased risk for anencephaly, and a 3.71-fold increased risk for spina bifida [49]. PAHs are an important class of environmentally prevalent xenobiotics that can activate oxidative and electrophilic signaling pathways in lymphoid and non-lymphoid cells, including myeloid, epithelial, and other cells [50]. Beside toxicity and mutagenicity $[23,51]$, PAHs, including the non-classified PAHs such as phenanthrene, are also known to cause immunotoxic effects, particularly IgE regulation [52].

In conclusion, millions of people worldwide have tattoos, for which high amounts of tattoo colorants are injected into the human body. A recent survey revealed that $28 \%$ of tattooed individuals have more than four tattoos and that $36 \%$ have tattoos that are larger than $900 \mathrm{~cm}^{2}$, implying that several grams of tattoo colorants have been injected into skin. Any systemic effects of tattoo colorants, which are administered via tattooing, have not been investigated; thus, we highly recommend performing pharmacological, toxicological and epidemiological studies to clarify the possible impact of tattooing on human health.

\section{References}

1 Dorfer L, Moser M, Bahr F, Spindler K, Egarter-Vigl E, Giullen S, Dohr G, Kenner T: A medical report from the stone age? Lancet 1999;354:1023-1025.

-2 Ortiz AE, Alster TS: Rising concern over cosmetic tattoos. Dermatol Surg 2012; 38:424-429.

-3 Soran A, Kanbour-Shakir A, Bas O, Bonaventura M: A tattoo pigmented node and breast cancer. Bratisl Lek Listy 2014;115:311-312.
4 Balasubramanian I, Burke JP, Condon E: Painful, pigmented lymphadenopathy secondary to decorative tattooing. Am J Emerg Med 2013;31:1001.e1-e2.

5 Yactor AR, Michell MN, Koch MS, Leete TG, Shah ZA, Carter BW: Percutaneous tattoo pigment simulating calcific deposits in axillary lymph nodes. Proc (Bayl Univ Med Cent) 2013;26:28-29.

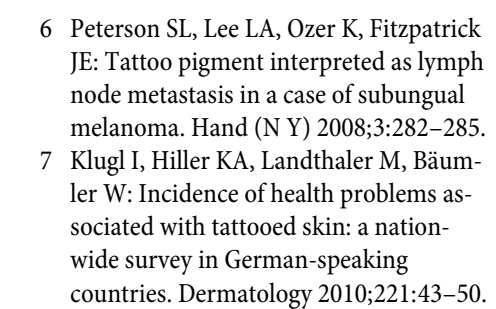

countries. Dermatology 2010;221:43-50. 
$>8$ Baumler W, Eibler ET, Hohenleutner U, Sens B, Sauer J, Landthaler M: Q-switch laser and tattoo pigments: first results of the chemical and photophysical analysis of 41 compounds. Lasers Surg Med 2000;26:13-21.

-9 Timko AL, Miller CH, Johnson FB, Ross $\mathrm{E}$ : In vitro quantitative chemical analysis of tattoo pigments. Arch Dermatol 2001; 137:143-147.

10 Papameletiou D, Zenie A, Schwela D, Bäumler W: Risks and health effects from tattoos, body piercing and related practices. http://gesundheit-nds.de/ downloads/risksandhealth.pdf, 2003 (accessed January 14, 2015).

11 Regensburger J, Lehner K, Maisch T, Vasold R, Santarelli F, Engel E, Gollmer A, Konig B, Landthaler M, Bäumler W: Tattoo inks contain polycyclic aromatic hydrocarbons that additionally generate deleterious singlet oxygen. Exp Dermatol 2010;19:e275-e281.

12 Wenzel SM, Rittmann I, Landthaler M, Bäumler W: Adverse reactions after tattooing: review of the literature and comparison to results of a survey. Dermatology 2013;226:138-147.

$\checkmark 13$ Serup J, Hutton Carlsen K: Patch test study of 90 patients with tattoo reactions: negative outcome of allergy patch test to baseline batteries and culprit inks suggests allergen(s) are generated in the skin through haptenization. Contact Dermatitis 2014;71:255-263.

14 Engel E, Spannberger A, Vasold R, Konig B, Landthaler M, Bäumler W: Photochemical cleavage of a tattoo pigment by UVB radiation or natural sunlight. J Dtsch Dermatol Ges 2007;5:583-589.

15 Cui Y, Spann AP, Couch LH, Gopee NV, Evans FE, Churchwell MI, Williams LD, Doerge DR, Howard PC: Photodecomposition of pigment yellow 74, a pigment used in tattoo inks. Photochem Photobiol 2004;80:175-184.

16 Vasold R, Naarmann N, Ulirch H, Fischer D, König B, Landthaler M, Bäumler W: Tattoo pigments are cleaved by laser light - the chemical analysis in vitro evidence for hazardous compounds. Photochem Photobiol 2004;80:185-190.

17 Huang QG, Kong LR, Liu YB, Wang LS: Relationships between molecular structure and chromosomal aberrations in in vitro human lymphocytes induced by substituted nitrobenzenes. Bull Environ Contam Toxicol 1996;57:349-353.
18 Shimizu H, Kumada T, Nakano S, Kiriyama S, Sone Y, Honda T, Watanabe K, Nakano I, Fukuda Y, Hayakawa T: Liver dysfunction among workers handling 5-nitro-o-toluidine. Gut 2002;50:266270.

19 Sayama M, Mori M, Shoji M, Uda S, Kakikawa M, Kondo T, Kodaira KI: Mutagenicities of 2,4- and 2,6-dinitrotoluenes and their reduced products in salmonella typhimurium nitroreductase- and o-acetyltransferaseoverproducing ames test strains. Mutat Res 1998;420:27-32.

20 National Toxicology Program: Toxicology and carcinogenesis studies of 1,4-dichlorobenzene (cas no. 106-46-7) in f344/n rats and b6c3f1 mice (gavage studies). Natl Toxicol Program Tech Rep Ser 1987;319:1-198.

21 Lo HH, Brown PI, Rankin GO: Acute nephrotoxicity induced by isomeric dichloroanilines in Fischer 344 rats. Toxicology 1990;63:215-231.

22 IARC: Carbon Black; in IARC Monographs on the Evaluation of Carcinogenic Risks to Humans. Volume 93: Carbon Black, Titanium Dioxide, and Talc. Lyon, International Agency for Research on Cancer, 2010. http://monographs. iarc.fr/ENG/Monographs/vol93/ mono93.pdf (accessed January 14, 2015).

23 IARC: IARC Monographs on the Evaluation of Carcinogenic Risks to Humans. Volume 92: Some Non-Heterocyclic Polycyclic Aromatic Hydrocarbons and Some Related Exposures. Lyon, International Agency for Research on Cancer, 2010. http://monographs.iarc.fr/ENG/ Monographs/vol92/mono92.pdf (accessed January 14, 2015).

24 Koreeda M, Moore PD, Wislocki PG, Levin W, Yagi H, Jerina DM: Binding of benzo[a]pyrene 7,8-diol-9,10-epoxides to DNA, RNA, and protein of mouse skin occurs with high stereoselectivity. Science 1978;199:778-781.

25 Newman MJ, Light BA, Weston A, Tollurud D, Clark JL, Mann DL, Blackmon JP, Harris CC: Detection and characterization of human serum antibodies to polycyclic aromatic hydrocarbon diol-epoxide DNA adducts. J Clin Invest 1988;82:145-153.

-26 Bizub D, Wood AW, Skalka AM: Mutagenesis of the ha-ras oncogene in mouse skin tumors induced by polycyclic aromatic hydrocarbons. Proc Natl Acad Sci U S A 1986;83:6048-6052.
27 Davila DR, Romero DL, Burchiel SW: Human $t$ cells are highly sensitive to suppression of mitogenesis by polycyclic aromatic hydrocarbons and this effect is differentially reversed by alpha-naphthoflavone. Toxicol Appl Pharmacol 1996;139:333-341.

28 van Grevenynghe J, Rion S, Le Ferrec E, Le Vee M, Amiot L, Fauchet R, Fardel O: Polycyclic aromatic hydrocarbons inhibit differentiation of human monocytes into macrophages. J Immunol 2003;170:2374-2381.

29 Lehner K, Santarelli F, Vasold R, Konig B, Landthaler M, Baumler W: Black tattoo inks are a source of problematic substances such as dibutyl phthalate. Contact Dermatitis 2011;65:231-238.

-30 Hogsberg T, Loeschner K, Lof D, Serup $\mathrm{J}$ : Tattoo inks in general usage contain nanoparticles. Br J Dermatol 2011;165: 1210-1218.

31 Zhang M, Jin J, Chang YN, Chang X, Xing G: Toxicological properties of nanomaterials. J Nanosci Nanotechnol 2014;14:717-729.

32 Varga E, Korom I, Varga J, Kohan J, Kemeny L, Olah J: Melanoma and melanocytic nevi in decorative tattoos: three case reports. J Cutan Pathol 2011;38: 994-998.

-33 Kluger N, Koljonen V: Tattoos, inks, and cancer. Lancet Oncol 2012;13:e161-e168.

34 Engel E, Santarelli F, Vasold R, Maisch T, Ulrich H, Prantl L, Konig B, Landthaler M, Baumler W: Modern tattoos cause high concentrations of hazardous pigments in skin. Contact Dermatitis 2008; 58:228-233.

35 Ferguson JE, Andrew SM, Jones CJ, August PJ: The q-switched neodymium:Yag laser and tattoos: a microscopic analysis of laser-tattoo interactions. Br J Dermatol 1997;137:405-410.

-36 Lehner K, Santarelli F, Penning R, Vasold R, Engel E, Maisch T, Gastl K, Konig B, Landthaler M, Bäumler W: The decrease of pigment concentration in red tattooed skin years after tattooing. J Eur Acad Dermatol Venereol 2011;25:1340-1345.

- 37 Engel E, Vasold R, Santarelli F, Maisch T, Gopee NV, Howard PC, Landthaler M, Bäumler W: Tattooing of skin results in transportation and light-induced decomposition of tattoo pigments - a first quantification in vivo using a mouse model. Exp Dermatol 2010;19:54-60. 
38 Gelboin HV: Benzo[alpha]pyrene metabolism, activation and carcinogenesis: role and regulation of mixed-function oxidases and related enzymes. Physiol Rev 1980;60:1107-1166.

39 Moraitis AG, Hewison M, Collins M, Anaya C, Holick MF: Hypercalcemia associated with mineral oil-induced sclerosing paraffinomas. Endocr Pract 2013;19:e50-e56.

40 Akintobi AM, Villano CM, White LA: 2,3,7,8-tetrachlorodibenzo-p-dioxin (tcdd) exposure of normal human dermal fibroblasts results in ahr-dependent and -independent changes in gene expression. Toxicol Appl Pharmacol 2007; 220:9-17.

41 Shimada T, Fujii-Kuriyama Y: Metabolic activation of polycyclic aromatic hydrocarbons to carcinogens by cytochromes p450 la1 and 1b1. Cancer Sci 2004;95: $1-6$.

42 Buters JT, Sakai S, Richter T, Pineau T, Alexander DL, Savas U, Doehmer J, Ward JM, Jefcoate CR, Gonzalez FJ: Cytochrome 450 cyp 1 b1 determines susceptibility to 7, 12-dimethylbenz[a]anthracene-induced lymphomas. Proc Natl Acad Sci U S A 1999;96:1977-1982.
43 Kaur RR, Kirby W, Maibach H: Cutaneous allergic reactions to tattoo ink. J Cosmet Dermatol 2009;8:295-300.

44 Engel E, Ulrich H, Vasold R, Konig B, Landthaler M, Suttinger R, Baumler W: Azo pigments and a basal cell carcinoma at the thumb. Dermatology 2008;216: 76-80.

-45 Jaigirdar AA, Yeh MW, Sharifi E, Browne LW, Leong SP: Coexisting tattoo pigment and metastatic melanoma in the same sentinel lymph node. J Cutan Med Surg 2009;13:321-325.

46 Dominguez E, Alegre V, Garcia-Melgares ML, Laguna C, Martin B, Sanchez JL, Oliver V: Tattoo pigment in two lymph nodes in a patient with melanoma. J Eur Acad Dermatol Venereol 2008;22:101102.

47 Honegger MM, Hesseltine SM, Gross JD, Singer C, Cohen JM: Tattoo pigment mimicking axillary lymph node calcifications on mammography. AJR Am J Roentgenol 2004;183:831-832.
48 Schlager A, Laser A, Melamed J, Guth AA: A tattoo-pigmented node masquerading as the sentinel node in a case of breast cancer. Breast J 2008;14:299-300.

49 Ren A, Qiu X, Jin L, Ma J, Li Z, Zhang L, Zhu H, Finnell RH, Zhu T: Association of selected persistent organic pollutants in the placenta with the risk of neural tube defects. Proc Natl Acad Sci U S A 2011;108:12770-12775.

50 Burchiel SW, Luster MI: Signaling by environmental polycyclic aromatic hydrocarbons in human lymphocytes. Clin Immunol 2001;98:2-10.

51 EC-SCF: Polycyclic aromatic hydrocarbons - occurrence in foods, dietary exposure and health effects. SCF/CS/ CNTM/PAH/29 ADD1. 2002. http://ec. europa.eu/food/fs/sc/scf/out154_en.pdf (accessed January 14, 2015).

52 Tsien A, Diaz-Sanchez D, Ma J, Saxon A: The organic component of diesel exhaust particles and phenanthrene, a major polyaromatic hydrocarbon constituent, enhances ige production by ige-secreting ebv-transformed human $b$ cells in vitro. Toxicol Appl Pharmacol 1997;142:256-263. 